\title{
Thymoma-Associated Progressive Encephalomyelitis with Rigidity and Myoclonus Successfully Treated with Thymectomy and Intravenous Immunoglobulin
}

\author{
Taira Uehara Hiroyuki Murai Ryo Yamasaki Hitoshi Kikuchi Hiroshi Shigeto \\ Yasumasa Ohyagi Jun-ichi Kira \\ Department of Neurology, Neurological Institute, Graduate School of Medical Sciences, Kyushu University, \\ Fukuoka, Japan
}

\begin{abstract}
Dear Sir,
Progressive encephalomyelitis with rigidity and myoclonus (PERM) is a rare central nervous system disorder that is characterized by systemic muscular rigidity, painful spasms, pathological reflexes, myoclonus and neuropsychological alterations [1]. Here, we report a case of thymoma-associated PERM which was successfully treated with thymectomy followed by repetitive intravenous immunoglobulin (IVIG) therapy.
\end{abstract}

\section{Case Report}

A 52-year-old woman began to feel awkwardness in her left leg in February 2004. One month later she had difficulty walking because of stiffness of her left leg. Gradually, stiffness also occurred in her right leg and finally she was unable to stand up. She began to experience painful spasms of the lower extremities. She received intravenous injection of methylprednisolone and double-filtration plasmapheresis at another hospital. Despite some limited improvement in the painful spasms, these treatments did not alleviate her symptoms. She was referred to our hospital for further treatment in April 2004.

On admission, she was alert, but her mood was irritable and she had anxiety. Cranial nerves were normal. Limb and trunk muscles were stiff and painful mus- cle spasms were frequently experienced. Her neck, trunk and extremities showed rigido-spasticity. Painful spasms were easily elicited by auditory, visual and tactile stimuli. Reflex myoclonus was also observed. Hyper-reflexia was seen in 4 limbs and pathological reflexes were bilaterally positive. Sensation was normal. Blood cell counts and serum chemistry revealed no abnormalities. The anti-glutamic acid decarboxylase (GAD) antibody level was $9,020 \mathrm{U} / \mathrm{ml}$ in serum and $109 \mathrm{U} / \mathrm{ml}$ in cerebrospinal fluid (normal $\leq 1.5$ ). Anti-acetylcholine receptor antibody was positive at a titer of $11.9 \mathrm{nmol} / \mathrm{l}$ (normal $\leq 0.2$ ). Serum anti-amphiphysin antibody was negative. Cerebrospinal fluid analysis showed no abnormality. Head and spinal cord MRI was normal. There was no slowing in electroencephalography. Nerve conduction study was normal. There was no decrease in compound muscle action potential on repetitive stimulation of the median nerve. Needle electromyography revealed continuous contraction of both agonist and antagonist muscles in the extremities (fig. 1A). Thoracic $\mathrm{CT}$ depicted the presence of thymoma in the anterior mediastinum (fig. 1B).

\section{Clinical Course}

Simple plasma exchanges were performed 6 times over a period of 4 weeks. Despite reduction in the serum anti-GAD antibody level to $6,090 \mathrm{U} / \mathrm{ml}$, her muscle stiffness became worse and she could hardly roll over in May 2004. Stiffness also spread to the facial and oropharyngeal muscles and her phonation became feeble. Therefore, we performed thymectomy in June 2004. The surgical specimen was composed of a proliferation of mature lymphocytes, and was sparsely distributed with epithelioid cells (WHO type B1; fig. 1C). After the operation, clinical symptoms started to improve. One month after surgery, muscle stiffness had reduced to some extent, but her movement was still severely impaired. We therefore treated her with 3 courses of IVIG over 3 months. Each course consisted of IVIG at a dose of $400 \mathrm{mg} / \mathrm{kg} /$ day for 5 consecutive days. Along with the treatment, her muscle stiffness improved gradually, and she could maintain a sitting position in September. Her mood also improved. In November 2004 , she was able to walk with a stick. Seven months after the operation, she could care for herself without assistance, and the Babinski sign was no longer positive. She fully returned to her ordinary daily living, which has now persisted for $>7$ years, without relapse or deterioration.

\section{Paired Transcranial Magnetic}

Stimulation

Excitability of the motor cortex was evaluated with transcranial magnetic stimulation (TMS) using the paired-pulse para-

\section{KARGER}

Fax +4161306 1234 E-Mail karger@karger.ch www.karger.com www.karger.com/ene
Dr. H. Murai

Department of Neurology, Iizuka Hospital

3-83 Yoshio-machi, Iizuka 820-8505 (Japan)

Tel. +81948223800

E-Mailhmurai@neuro.med.kyushu-u.ac.jp 

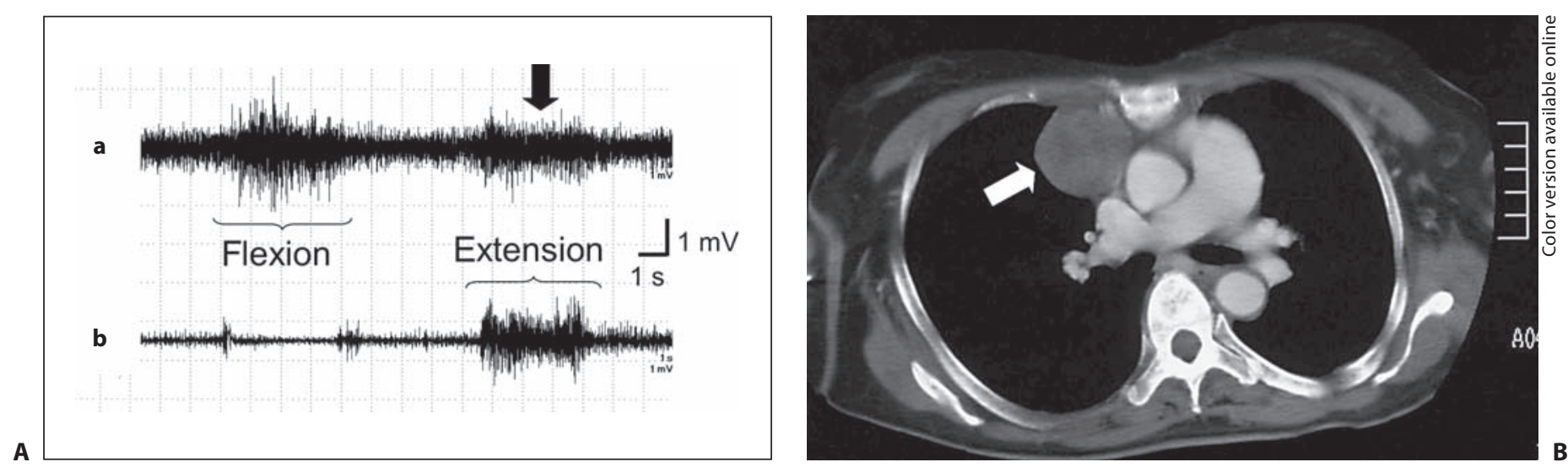

Fig. 1. A Needle electromyography of the left biceps brachii muscle (a) and the left triceps brachii muscle (b), which showed cocontraction of both agonist and antagonist muscles (arrow). B Chest computed tomography scan revealed thymoma (arrow) in the anterior mediastinum. C Pathological findings demonstrated that thymoma is mainly composed of the proliferation of mature lymphocytes and sparsely distributed round-polygonal epithelioid cells.

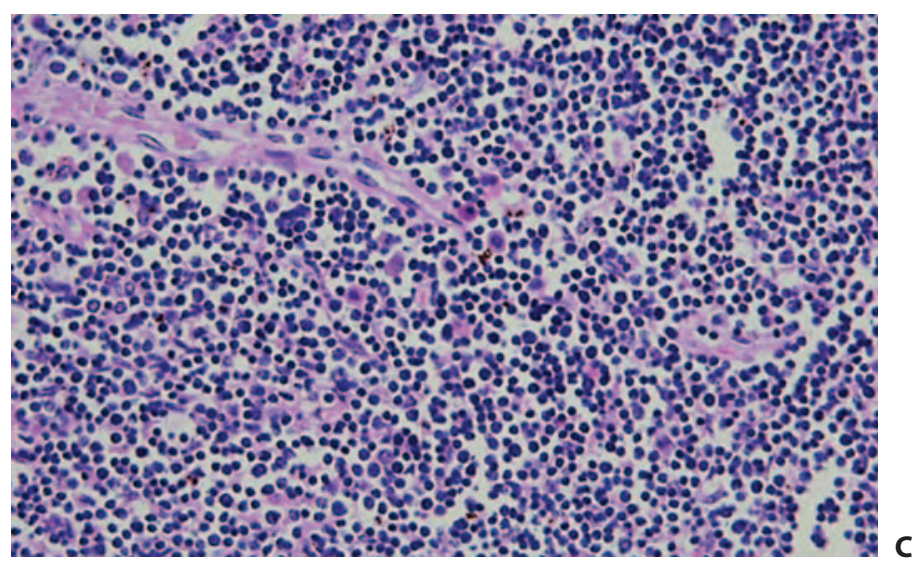

Fig. 2. Paired transcranial magnetic stimulation was performed before (dotted bold line) and after treatment (solid bold line) on the present patient and healthy controls (solid thin line). Intracortical inhibition at the interstimulus intervals of $1 \mathrm{~ms}$ was insufficient before treatment, and it was normalized to a level comparable to healthy controls after thymectomy and IVIG. MEP $=$ Motor evoked potential.

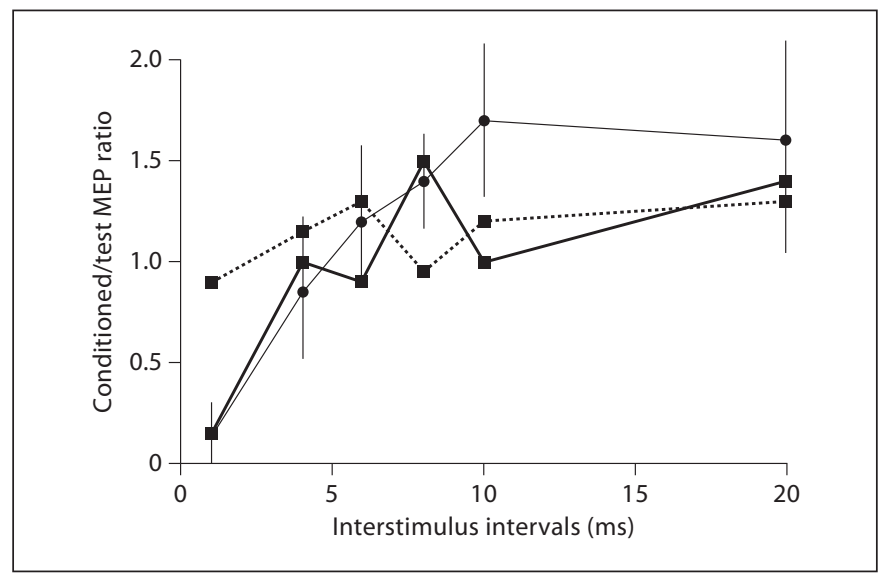

digm, as described previously [2]. TMS was delivered on the motor cortex, and motor evoked potentials were recorded in the right abductor pollicis brevis muscle. Interstimulus intervals were 1,4 and $6 \mathrm{~s}$, and 8 , 10 and $20 \mathrm{~ms}$. The ratio of conditioned and test motor evoked potentials was calculated. Paired TMS was performed before and after treatment of the present patient and in healthy controls $(n=6$, age $27.0 \pm 2.0$ years). The results suggested that intracortical inhibition of the present patient at an interstimulus interval of $1 \mathrm{~ms}$ was insufficient before treatment, and that it normalized to a level comparable to healthy controls after thymectomy and IVIG (fig. 2).

\section{Discussion}

There is controversy as to whether PERM and stiff-person syndrome (SPS) share the same disease spectrum. Brown and Marsden [3] distinguished PERM from SPS using the term 'stiff-man plus syndrome'. Treatment response of PERM 
is poor and the disease relentlessly progresses and leads to death. PERM is sometimes associated with neoplasms. There has been a report of lung cancer-associated paraneoplastic PERM [4], and 1 case with thymoma [5].

In the present case, paired TMS clearly demonstrated the cortical involvement of the disease, given that motor cortex hyperexcitability was alleviated by treatment. Various neuronal autoantibodies can be detected in thymoma patients [6]. Interestingly, there is a report of encephalopathy which occurred in a thymoma-associated myasthenia gravis patient in whom anti- acetylcholine receptor antibody was also detected in cerebrospinal fluid [7]. It is possible that thymoma participates in the process of autoimmunity against various neuronal autoantigens. Therefore, in the present case, some thymoma-associated neuronal autoantibodies besides antiGAD antibody might be associated with the addition of cortical involvement.

Another notable feature of the present case was the excellent response to thymectomy and IVIG. Among the reported cases of thymoma-associated SPS, thymectomy was performed in 3 cases and was reported to be effective in 2 cases $[8,9]$, although in
1 case, the effects on SPS was not reported [10]. The beneficial effects of IVIG on SPS have been described in the literature $[1,11]$. With regard to PERM, plasmapheresis and corticosteroids were reportedly effective in 1 case [12]. Thymectomy was reported to be effective only in a case of thymomaassociated PERM [5]. In our case, the effects of thymectomy alone were not sufficient enough and the patient required 3 courses of IVIG. Therefore, repeated IVIG should be considered after thymectomy in refractory cases with thymoma-associated PERM.

\section{References}

1 Meinck HM, Thompson PD: Stiff man syndrome and related conditions. Mov Disord 2002;17:853-866.

- 2 Koerner C, Wieland B, Richter W, Meinck HM: Stiff-person syndromes: motor cortex hyperexcitability correlates with anti-GAD autoimmunity. Neurology 2004;62:13571362.

-3 Brown P, Marsden CD: The stiff man and stiff man plus syndromes. J Neurol 1999;246: 648-652.

-4 Spitz M, Ferraz HB, Barsottini OG, Gabbai AA: Progressive encephalomyelitis with rigidity: a paraneoplastic presentation of oat cell carcinoma of the lung. Case report. Arq Neuropsiquiatr 2004;62:547-549.
-5 Clerinx K, Breban T, Schrooten M, Leite MI, Vincent A, Verschakelen J, Tousseyn T, Vandenberghe W: Progressive encephalomyelitis with rigidity and myoclonus: resolution after thymectomy. Neurology 2011;76:303-304.

6 Vernino S, Lennon VA: Autoantibody profiles and neurological correlations of thymoma. Clin Cancer Res 2004;10:7270-7275.

-7 Bogousslavsky J, Regli F, Doret AM, Fulpius BW, Ostinelli B, Rabinowicz T, Ruzicka J: Encephalopathy, peripheral neuropathy, dysautonomia, myasthenia gravis, malignant thymoma, and antiacetylcholine receptor antibodies in the CSF. Eur Neurol 1983; 22:301-306.

8 Nicholas AP, Chatterjee A, Arnold MM, Claussen GC, Zorn GL Jr, Oh SJ: Stiff-persons' syndrome associated with thymoma and subsequent myasthenia gravis. Muscle Nerve 1997;20:493-498.
9 Hagiwara H, Enomoto-Nakatani S, Sakai K, Ugawa Y, Kusunoki S, Kanazawa I: Stiff-person syndrome associated with invasive thymoma: a case report. J Neurol Sci 2001;193: 59-62.

10 Thomas S, Critchley P, Lawden M, Farooq S, Thomas A, Proudlock FA, Constantinescu CS, Gottlob I: Stiff person syndrome with eye movement abnormality, myasthenia gravis, and thymoma. J Neurol Neurosurg Psychiatry 2005;76:141-142.

11 Dalakas MC, Fujii M, Li M, Lutfi B, Kyhos J, McElroy B: High-dose intravenous immune globulin for stiff-person syndrome. N Engl J Med 2001;345:1870-1876.

12 Fogan L: Progressive encephalomyelitis with rigidity responsive to plasmapheresis and immunosuppression. Ann Neurol 1996;40: 451-453. 